\title{
Insulin receptor and insulin-responsive glucose transporter (GLUT 4) mutations and polymorphisms in a Welsh Type 2 (non-insulin-dependent) diabetic population
}

\author{
S.O'Rahilly ${ }^{1}$, A.Krook ${ }^{1}$, R.Morgan ${ }^{2}$, A. Rees ${ }^{2}$, J.S.Flier ${ }^{3}$ and D.E.Moller ${ }^{3}$ \\ ${ }^{1}$ Departments of Medicine and Clinical Biochemistry, University of Cambridge, Addenbrooke's Hospital, Cambridge, ${ }^{2}$ Department of \\ Medicine, University Hospital of Wales, Cardiff, UK and ${ }^{3}$ Department of Medicine, Beth Israel Hospital, Boston, Massachusetts, USA
}

\begin{abstract}
Summary. We have recently examined the exons encoding the insulin receptor tyrosine kinase domain and GLUT 4 in 30 subjects with Type 2 (non-insulin-dependent) diabetes mellitus using a molecular scanning approach. The variant sequences Val-Met ${ }^{985}$ and Lys-Glu ${ }^{1068}$ of the insulin receptor and Val-Ile ${ }^{383}$ of GLUT 4 were each separately found in three different diabetic subjects. In a study of a Welsh population, the GLUT $4^{383}$ variant was found in three of 160 diabetic and none of the 80 control subjects. In this study, the same group of Welsh Type 2 diabetic and control subjects was analysed using allele-specific oligonucleotide hybridisation, single nucleotide primer extension and allele-specific restriction digestion to ascertain the frequency of the two insulin receptor mutations. The Val-Met ${ }^{335}$ mutation was found in none of the 160 Welsh Caucasian Type 2 diabetic subjects and two of 80 control subjects. The Lys-Glu ${ }^{1068}$ mutation removes a Sty 1 site and digestion of amplified exon 18 with Sty 1 confirmed the presence of this mutation in the heterozygous state in the original subject. None of the Welsh diabetic or control sub-
\end{abstract}

jects had the Glu ${ }^{1068}$ mutation. The discovery of a very common silent polymorphism at codon 130 of GLUT 4 allowed examination of the association of this locus with Type 2 diabetes using allele-specific oligonucleotide hybridisation in a subset of the Welsh subjects. The genotypic frequencies (homozygous wild-type and heterzygous polymorphic (poly) sequences) were not significantly different between diabetic and control subjects (Type 2 diabetic subjects: wildtype/wild-type $40 \%$, wild-type/poly $46 \%$, poly/poly $14 \%$; Control subjects: wild-type/wild-type $37 \%$, wild-type/poly $45 \%$, poly/poly $18 \% ; p>0.05$ ). In conclusion, in a British Caucasian population the examined insulin receptor tyrosine kinase domain mutations are uncommon. Also the GLUT 4 locus does not appear to be strongly associated with Type 2 diabetes.

Key words: Genetics, Type 2 (non-insulin-dependent) diabetes mellitus, insulin receptor, glucose transporters.
Recently, several subjects with syndromes of severe insulin resistance such as leprechaunism, Rabson Mendenhall syndrome and type $A$ insulin resistance have been found to have insulin receptor mutations [1]. In certain cases heterozygous relatives of such patients have had no obvious clinical features except moderate to marked hyperinsulinaemia and sometimes, impairment of glucose tolerance [2]. This has led to increased interest in the possibility that a certain proportion of subjects with "typical" Type 2 (non-insulin-dependent) diabetes mellitus may harbour such mutations and that these might contribute to the inherited insulin resistance component of the disease. Studies of the insulin receptor locus in Type 2 diabetes and control populations by restriction fragment length polymorphisms (RFLP) have produced conflicting results while the sequence of the receptor has been found to be normal in the small number of Type 2 diabetic patients in whom it has been established [3]. We have recently employed a "molecular scanning" technique, namely single stranded conformation polymorphism (SSCP) analysis, for the analysis of the insulin receptor and insulin-responsive glucose transporter genes in Type 2 diabetic patients $[4,5]$. We established that the method has a high specificity and sensitivity for the detection of variant sequences and, when applied to the screening of 30 Caucasian Type 2 diabetic subjects, it detected two novel insulin receptor variants. The first aim of this study was to establish the frequency of these variants in a large well-matched group of Type 2 diabetic and control subjects in order to assess the potential importance of these variant receptors to the larger Type 2 diabetic population.

In a prior study of the GLUT 4 locus by SSCP we detected a common silent polymorphism at codon 130 in exon 4 a [5]. As RFLP's of GLUT 4 have not been frequent or informative, and as preliminary data from other 
A

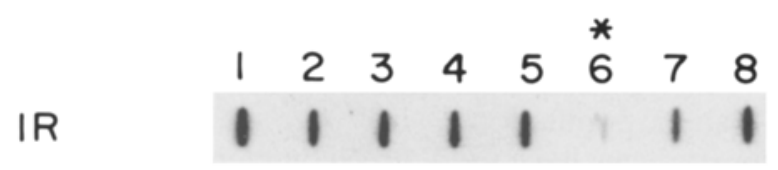

IR-985
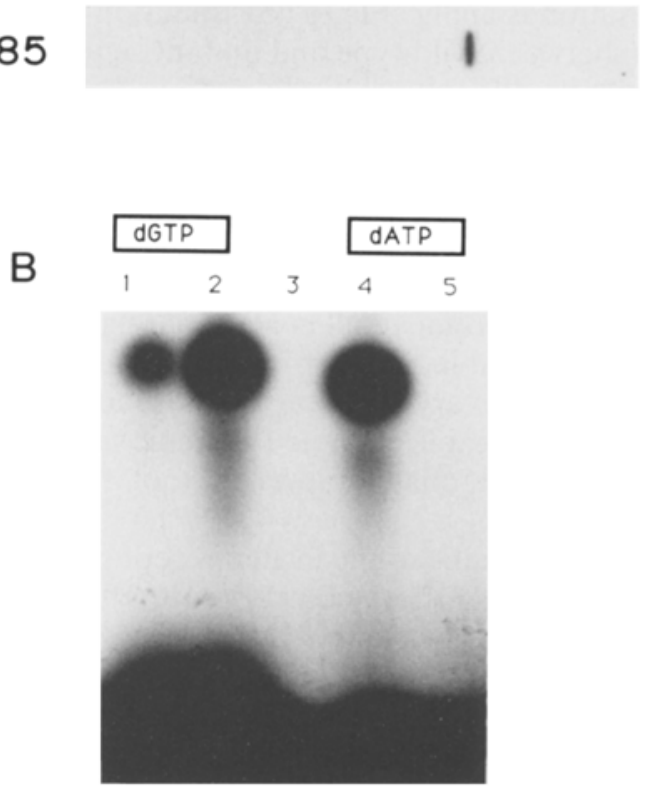

Fig.1. A Allele specific oligonucleotide (ASO) hybridisation of wild-type (above) and mutant (below) probes for the detection of insulin receptor (IR) mutant Val (GTG)-Met (ATG) ${ }^{985}$. Subject 6 is a heterozygote and other subjects are homozygous for the wild-type sequence. B Single nucleotide primer extension (SNuPE) analysis of the insulin receptor 985 mutant. Lane 3 is blank. Lanes 1 and 4 contain amplified exon 17 from a subject heterozygous for the $G$ to A mutation at the 985 locus. Lanes 2 and 5 contain amplified exon 17 from a subject homozygous for the wild-type sequence. Lanes 1 and 2 show incorporation of the ${ }^{32} \mathrm{P}$-labelled wild-type nucleotide $(\mathrm{G})$ in both subjects after the SNuPE reaction. Lane 4 shows incorporation of the ${ }^{32}$ P-labelled mutant nucleotide (A) in the heterozygous subject but not in the homozygous wild-type subject in lane 5

workers suggested an association of a marker in the exon 3-5 region of GLUT 4 with Type 2 diabetes [6] we used allele-specific oligonucleotide (ASO) hybridisation to examine the frequency of the polymorphic sequence in a subset of the Welsh Type 2 diabetic and control populations.

\section{Subjects, materials and methods}

The subjects studied by ASO and allele specific restriction fragment length polymorphism (ASRFLP) analysis were all Welsh Caucasians recruited at the University Hospital of Wales. Subjects gave informed consent to the studies. Diabetic subjects $(n=165)$ conformed to World Health Organisation (WHO) criteria. Normal subjects $(n=85)$ had no family history of diabetes and had normal random blood glucose levels (WHO criteria) and $\mathrm{HbA}_{1}$ less than $8.5 \%$. Mean age was $64.9 \pm 9.5$ years and mean body mass index was $24.6 \pm 4.5 \mathrm{~kg} / \mathrm{m}^{2}$.
Allele specific oligonucleotide hybridization

ASO was performed as previously described [5] using the following probes: for insulin receptor codon 985: wild-type - TGTGTAACG TGCCGGAC; mutant - TGTGTAACATGCCGGAC: for insulin receptor codon 1068: wild type - TGGTGTCCAAGGGCCAG; mutant - TGGTGTCCGAGGGCCAG: for GLUT 4 codon 130: wild-type - CCTGGCCAACGCTGGTG; mutant - CCTGG CCATGCTGGTG.

\section{Allele specific restriction digestion}

The Lys-Glu ${ }^{1068}$ mutation results in the loss of a Sty 1 site in exon 18. Exon 18 of the insulin receptor was amplified from $100 \mathrm{ng}$ of genomic DNA from each subject using the polymerase chain reaction (PCR) as previously described [5]. Restriction digests were performed at $37^{\circ} \mathrm{C}$ for $3 \mathrm{~h}$ using $18 \mu \mathrm{l}$ of the PCR reaction and $10 \mathrm{U}$ of Sty 1 with the $\mathrm{NaCl}$ concentration adjusted to $0.1 \mathrm{~mol} / \mathrm{I}$. Ten $\mu$ of the unpurified digest was subjected to electrophoresis on a $1.7 \%$ agarose minigel at $150 \mathrm{~V}$ for $15 \mathrm{~min}$ and the DNA bands were visualisized by staining with ethidium bromide.

\section{Single nucleotide primer extension ( $S N u P E$ )}

A 17-mer oligonucleotide (TTCCATGCTCTGTGTAC) was designed which hybridised to the 17 nucleotides immediately $5^{\prime}$ to the site of the GTG-ATG mutation in codon 985 of the insulin receptor. Fifty nanograms of amplified exon 17 from three subjects with the wild-type sequence only and three subjects who were heterozygous for the mutant sequence by ASO, were examined. Using amplified exon 17 material as template, duplicate SNuPE reactions were set up for each subject as described above for $\mathrm{PCR}$ reactions except for the absence of deoxynucleotide tri-phosphates (dNTPs) and the presence in one tube of $\alpha{ }^{32}$ P-labelled deoxy ATP (wild-type) and the presence in the other tube of $\alpha_{-}{ }^{32} \mathrm{P}-$ labelled deoxy GTP (mutant). The samples were subjected to one cycle consisting of 2 min denaturation at $94^{\circ} \mathrm{C} .2 \mathrm{~min}$ annealing at $55^{\circ} \mathrm{C}$ and 2 min primer extension at $72^{\circ} \mathrm{C}$. Five microlitres of sample was mixed with $5 \mathrm{ml}$ of gel loading buffer $(80 \%$ formamide $/ 50 \mathrm{mmol} / \mathrm{l}$ Tris Borate $\mathrm{pH} 8.3 / 1 \mathrm{mmol} / 1$ EDTA, $0.1 \%$ bromophenol blue) heated for $2 \mathrm{~min}$ at $90^{\circ} \mathrm{C}$ and loaded onto a $6 \%$ polyacrylamide gel containing $8 \mathrm{~mol} / \mathrm{l}$ urea. Gels were run for $2 \mathrm{~h}$ at $250 \mathrm{~V}$ and then subjected to autoradiography using Kodak X-AR5 film and exposed for $1-2 \mathrm{~h}$.

\section{Statistical analysis}

Statistical analysis of the GLUT 4 polymorphism data was performed using the Chi-square test.

\section{Results}

\section{Insulin receptor mutations}

The $\mathrm{Met}^{985}$ variant was detected by ASO analysis (Fig. 1 a) in two of 85 control subjects and none of the 160 Welsh diabetic subjects. Both subjects were heterozygotes. Single nucleotide primer extension was used to confirm the results of the ASO studies. Gel-purified exon 17 from the index patient and the two control subjects with the mutation were studied in parallel with three subjects in whom the ASO studies revealed only wild-type sequence. SNuPE analysis confirmed the ASO findings (Fig. 1 b). 


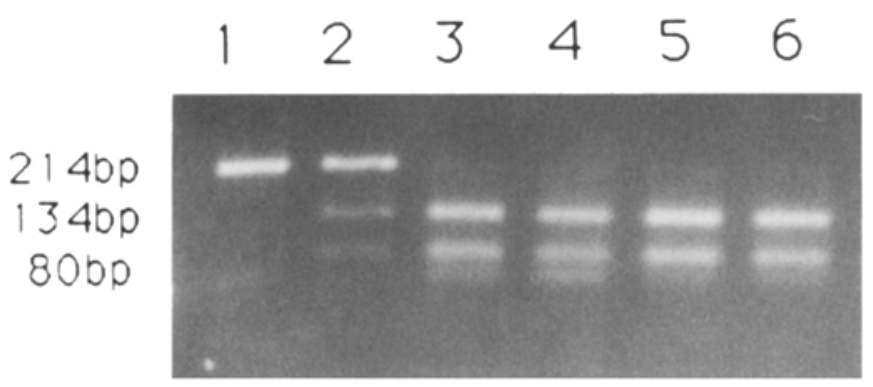

Fig. 2. Allele specific restriction digestion to detect the exon $18 \mathrm{in}$ sulin receptor mutation Lys-Glu ${ }^{1068}$. Polymerase chain reaction (PCR) amplification of exon 18 results in a 214 base pair (bp) fragment (Lane 1). Digestion of the wild-type PCR product with Sty 1 normally results in two fragments of 134 and $80 \mathrm{bp}$. The 1068 mutation removes the Sty 1 site and in a heterozygous individual (lane 2) three bands are seen, as a result of digestion of the amplified wildtype allele only

The Lys-Glu ${ }^{1068}$ mutation in the index case resulted in the expected ASRFLP pattern with the 314 base pair (bp) band representing the mutant allele and the 214 and $70 \mathrm{bp}$ bands representing the wild-type allele (Fig.2). This mutation was not detected in any of the Welsh diabetic or control subjects.

\section{GLUT 4 polymorphism}

A randomly selected subgroup of diabetic $(n=86)$ and control $(n=76)$ subjects were studied for the presence of the silent polymorphic variant in codon 130 of GLUT 4, exon $4 \mathrm{a}$. ASO hybridisation distinguished between subjects who were known to have homozygous wild-type (wt) and homo- and heterozygous polymorphic (poly) sequences. The genotype frequencies in both the diabetic and control populations did not deviate significantly from Hardy-Weinberg equilibrium. There was no significant difference in allelic (number of polymorphic alleles in Type 2 diabetic vs control subjects: $64 / 172$ vs $62 / 152$ : NS) or genotype frequencies (Type 2 diabetic subjects: wt/wt $34 / 86$, wt/poly $40 / 86$, poly/poly 12/86; Control subjects: wt/wt 28/76, wt/poly 34/76, poly/poly $14 / 76$; NS) between diabetic and control populations.

\section{Discussion}

The development of PCR-based techniques such as direct sequencing and SSCP analysis has facilitated the analysis of the fine structure of candidate genes in Type 2 diabetes and other disorders. Having used these techniques to detect a variant sequence in an affected subject, the relationship of the mutation to the disease remains to be established. Studies of the co-segregation of the mutation with metabolic abnormalities in families members and examination of the functional properties of the variant molecule when expressed in vitro are important steps in this evaluation. As Type 2 diabetes is likely to be a complex polygenic disorder and the metabolic alterations induced by one variant molecule in isolation may be subtle, studies of the prevalence of the mutation in large populations of Type 2 diabetic and control subjects are helpful in the assessment of its potential pathogenic role. In addition, this type of analysis can also provide information regarding the potential significance of this mutation for the broader Type 2 diabetic population.

In this study we have used three complementary techniques for the detection of known mutations. ASO hybridisation is applicable to any mutation but clear distinction between wild-type and mutant sequence can occasionally be difficult when the melting temperatures of the wild-type and mutant probes are similar. ASRFLP analysis is simpler and quicker, but is only applicable when the mutation results in a change in a convenient restriction site. The recently developed technique of SNuPE [7] requires the time consuming step of gel-purification of each PCR product to remove all contaminating dNTPs and is therefore unsuitable, in this form, for large-scale population studies. It is very useful as a confirmatory technique and may be applicable to pooled genomic samples thereby making it a potentially powerful tool for population genetic studies.

The $\mathrm{Val}^{985}$ residue of the insulin receptor is highly conserved between species and is also present in the analogous position in the insulin-like growth factor I receptor [8]. The variant residue $\mathrm{Met}^{985}$ represents a conservative amino acid substitution. Its presence in two elderly nondiabetic subjects suggests that it is unlikely to cause significant disturbance of glucose metabolism. In addition we have recently detected this variant in a young normoglycaemic subject with normal fasting insulin levels (unpublished observations). It seems likely that this is a normal variant of the insulin receptor, although it remains possible that, in combination with other genetic or environmental factors, it may contribute in some way to the glucose intolerance of the one diabetic patient carrying the mutation.

Lysine $^{1068}$ is also highly conserved and the Lys-Glu mutation is, a priori, more likely to be significant as it represents a change from a basic to an acidic amino acid. We detected this mutation solely in our index case and further family and in vitro studies will be necessary to establish its pathological significance.

Reduced insulin-mediated glucose disposal is a major feature of Type 2 diabetes and may be partially genetically determined [9]. GLUT 4 is therefore a strong candidate gene. One earlier study using denaturing gradient gel electrophoresis of genomic DNA had suggested an association between a polymorphism in the region of exon 3 to 5 of GLUT 4 and Type 2 diabetes in an American Caucasian population [6]. Other genetic studies to date have been limited because of the relative paucity of informative RFLPs. The finding of a common silent polymorphism at codon 130 enabled us to perform a simple case/control study which found no association between the polymorphism and Type 2 diabetes. The ability to detect this polymorphism by SSCP or ASO analysis will allow this to be a useful marker for linkage studies.

In conclusion we have applied a variety of methodologies to the assessment of the frequencies of specific insulin receptor tyrosine kinase domain mutations in a large 
population of Welsh Type 2 diabetic and control subjects and found these mutations to be uncommon. No association was found between a common silent polymorphism in exon $4 \mathrm{a}$ of GLUT 4 and Type 2 diabetes.

Acknowledgements. SO'R is a Wellcome Trust Senior Fellow in Clinical Science and was an MRC Travelling Fellow during some of these studies. We would like to thank Dr. W-H. Choi for his contributions to the study of the GLUT 4 gene and Drs. Graham Bell and John Buse for information regarding the genomic structure of GLUT4. The work was supported in part by a grant from the United States National Institutes of Health (NIDDK 280 82-10 JSF) and by a grant from the Juvenile Diabetes Foundation (DEM).

\section{References}

1. Taylor S, Kadowaki T, Kadowaki H, Accili D, Cama A, McKeon C(1990) Mutations in the insulin receptorgene in insulin-resistant patients. Diab Care 13: 257-279

2. Lekanne-Deprez RI, Potter van Loon BJ, van der Zon GCM et al. (1989) Individuals with only one allele for a functional insulin receptor have a tendency to hyperinsulinaemia but not to hyperglycaemia. Diabetologia 32: 740-744

3. Moller DE, Yokota A, Flier JS (1989) Normal insulin receptor cDNA sequence in Pima Indians with non-insulin dependent diabetes mellitus Diabetes 38: 1496-1500
4. O'Rahilly S, Choi WH, Patel P, Turner RC, Flier JS, Moller DE (1991) Detection of mutations in the insulin receptor gene in noninsulin-dependent diabetic patients by analysis of single-stranded conformation polymorphisms. Diabetes 40:777-782

5. Choi WH, O'Rahilly S, Rees A, Morgan R, Flier JS, Moller DE (1991) Molecular scanning of the insulin responsive glucose transporter gene in patients with non-insulin-dependent diabetes. Diabetes 40: 1712-1718

6. Goldfine A, Magre J, Goldstein BJ, Krowlewski AJ, Kahn CR (1991) Denaturing gradient gel electrophoresis of glucose transporter and insulin receptor genes. Clin Res 39: $383 \mathrm{~A}$ (Abstract)

7. Kuppuswamy MN, Hoffmann JW, Kasper CK, Spitzer SG, Groce SL, Bajaj SP (1991) Single nucleotide primer extension to detect genetic diseases: experimental application to haemophilia B and cystic fibrosis genes. Proc Natl Acad Sci USA 88: 1143-1147

8. Shier P, Watt VM (1989) Primary structure of a putative receptor for a ligand of the insulin family. J Biol Chem 264:14605-14608

9. Lillioja S, Mott DM, Zawadzki JK et al. (1987) In vivo insulin action is a familial characteristic in non-insulin dependent diabetic Pima Indians. Diabetes 36: 1329-1335

Received: 29 November 1991

and in revised form: 23 December 1991

Dr. S.O'Rahilly

Department of Medicine

Addenbrooke's Hospital

Cambridge CB22QR

UK 